\title{
Influence of biological and analytical variation on urine measurements for monitoring exposure to cadmium
}

\author{
H J Mason, N R Williams, M G Morgan, A J Stevenson, S Armitage
}

\begin{abstract}
Objective-To define the mean intraindividual biological variation in urinary concentrations of cadmium and retinol binding protein (RBP) in untimed, random urine samples and the influence of creatinine or specific gravity correction on reducing this variation. The relation between biological variation and analytical variation in defining uncertainty in a single measurement and significant differences between successive measurements was explored.

Methods-Repeat measurement study in subjects with either high historical exposure to cadmium but without current exposure, or unexposed volunteers. Standard statistical tools used in clinical laboratory medicine were applied to define intraindividual biological and analytical variation.

Results and conclusion-Both creatinine and specific gravity correction of urinary cadmium measurements in random urine samples seem to reduce the intraindividual variability compared with uncorrected values. With a standard definition, acceptable long term analytical precision for measurements of cadmium and RBP combined with creatinine analyses should be $<9 \%$ and $<15 \%$ respectively. The mean intraindividual biological variation of cadmium and RBP, expressed as creatinine corrected, was $18 \%$ and $40 \%$ respectively in the subjects exposed to cadmium. With the analytical precision used, significant differences $(p<0.05)$ between consecutive measurements for creatinine corrected urinary cadmium and RBP would need to show changes of $>54 \%$ and $>110 \%$ respectively. The relation between significant differences in consecutive results and differences in the analytical precision of the method used to measure the samples is described.
\end{abstract}

(Occup Environ Med 1998;55:132-137)

Keywords: urinary cadmium; urinary retinol binding protein; biological monitoring

Cadmium is a nephrotoxin with long half lives in the target organ and the liver, which is a major storage organ within the body. ${ }^{1}$ The renal effects occur above a kidney threshold concentration of the absorbed metal and are characterised by low molecular weight proteinuria. Evidence of a concommitant decrease in glomerular filtration rate or renal mass has been reported in subjects with proteinuria induced by cadmium. ${ }^{23}$ Exposure in industry has generally decreased over recent years but many workers have large body burdens of cadmium from historical exposure. For these workers, as well as any current exposure, there is the potential for transfer of cadmium from storage sites in the body to the kidney. Environmental exposure may also occur due to contaminated land from past industrial practices. ${ }^{4}$

Routine biological monitoring has used measurements of cadmium in blood and urine; the detection of renal effects can be made by the measurement of urinary retinol binding protein (RBP). In those chronically exposed, urinary cadmium has been reported to reflect body burden, or more particularly kidney burden of cadmium. ${ }^{5}$ Serial measurements of these variables in the occupational setting are used to monitor any build up of cadmium in a person and to ensure that concentrations are controlled to below the renal critical threshold. For practical purposes, measurements are usually performed in random, untimed, or spot urine samples corrected for urinary flow rate by creatinine concentration or specific gravity. Similar measurements have been used in epidemiological studies of the level of environmental cadmium contamination. ${ }^{4}$

The Employment Medical Advisory Service (EMAS) of the Health and Safety Executive (HSE), Birmingham had been monitoring for 12 years a cohort of workers with historical high exposure to cadmium from their use of cadmium/silver solders. A substantial proportion of the cohort has evidence of low molecular weight proteinuria, but their exposure to cadmium from solders had stopped totally or been reduced to a minimum for the 10 years before this study. The EMAS had noted a wide degree of variation in the quarterly measurements of urinary cadmium and RBP over the years in these people. This led to a questioning of the influence of this variation on interpretation of urinary cadmium and RBP as defining changes in cadmium burden or extent of renal damage. A study was initiated into defining the variability in these measurements and the influence of factors such as analytical precision, diurnal variation, and the use of creatinine correction on the measured intraindividual variation. Definition of these variables would aid in interpreting changes in serial measurements of urinary cadmium and RBP as indicating important 
changes in cadmium burden or renal function. It would also define the uncertainty associated with a single urinary measurement. Some years ago protocols were developed for investigating these fundamental characteristics of a measurement as an aid to diagnosis. ${ }^{67}$ Such methods, derived from clinical laboratory medicine, also allow for definition of acceptable laboratory analytical precision for the analyte of interest. ${ }^{8}$

This report details our investigation of intraindividual variation of urinary cadmium and RBP measured in spot urine samples with standard techniques used in clinical laboratory medicine.

Study design, methods, and subjects The basis of the study design is that if appropriate subjects give several samples of urine over the short time frame of a working week, the distribution of measurements in a subject's samples of urine will describe the true excretion in each person with an associated degree of uncertainty or variability. The spread of results in a person is a combination of both intraindividual biological variability and a component of error due to analytical imprecision in the laboratory. The half life of cadmium in the body and kidneys is between five and 30 years ${ }^{9}$ and thus in those subjects with no current exposure, multiple urine samples from a person over a short time frame should allow description of the variability in excretion of the metal. Likewise in those with low molecular weight proteinuria induced by cadmium any change in the degree of dysfunction is not discernible over a short time and multiple urine samples will reflect the variability of excretion of specific proteins.

Ten subjects with a history of chronic, high occupational exposure to cadmium, who were under continuing clinical investigation by EMAS, gave informed and written consent to their participation in the study after its purpose was explained to them. The exposure of these 10 workers to cadmium over the past 10 years had been minimal, but nine of the 10 workers were considered to have evidence of persistent low molecular weight proteinuria. These subjects had also been chronically exposed to silver. Five laboratory volunteers who had neither a history of occupational exposure to cadmium nor evidence of renal abnormality also gave their informed consent to taking part in the study. No restriction on dietary protein intake or exercise which may influence protein excretion was made on the volunteers during the study.

All subjects were asked to provide five random untimed urine samples throughout a single day. Time bands for collection of these spot urine samples were; time point $1=0600$ 0800 , time point $2=0800-1000$, time point $3=$ $1000-1200$, time point $4=1200-1400$, time point $5=1400-1600$. These time points include several suggested times for sample collection in occupational biological monitoring strategies - that is, first urine of the day, preshift, and during the shift.
On each of the other four days of the working week, subjects provided a single urine sample at 1000 . Therefore subjects provided a maximum number of nine samples during one week. All subjects, except one who had retired, were at work during the study, and several missing samples were recorded throughout the study. When appropriate, incomplete data sets were included in statistical analysis after weighting for the number of duplicate measurements for each person. ${ }^{7}$

Samples were transported on ice and stored frozen at $-70^{\circ} \mathrm{C}$ until analysis within one month of collection. Urine samples were randomised in large batches and duplicate measurements were performed for all analytes. Quality control material was analysed at regular intervals throughout the analytical batches. Urinary cadmium was analysed by inductively coupled plasma mass spectrometry (ICP-MS) and urinary retinol binding protein was analysed with an enzyme linked immunoassay. All are established methods in the Biomedical Sciences Group, Health and Safety Laboratory. Urinary specific gravity and creatinine, by alkaline picrate methodology, were also measured and used to correct analyte concentrations for the effects of urinary concentration or dilution. Current practise within the Health and Safety Laboratory disregards results in dilute urine samples which have a creatinine concentration $<3 \mathrm{mmol} / \mathrm{l}$. This criteria was applied to the study.

Statistical methods used to calculate intraindividual variation and analytical variation have been well documented ${ }^{7}$ and published in an occupational monitoring study. ${ }^{8}$ The influence of the time of sampling on analyte excretion was examined in the following manner. Each mean analyte result from the duplicate measurements at each of the five time points collected during the single day for a person exposed to cadmium was standardised to the mean concentration of all five results for that person. These standardised analyte results were used to calculate mean and distribution for each time point for the group and to compare time points with analysis of variance (ANOVA), linear trend over time, and Bonferroni's multiple range tests.

\section{Results}

The influence of the time of urine sampling on excretion of cadmium and RBP were studied in the samples from workers exposed to cadmium collected at varying times on a single day. The analyte concentrations in spot urine samples were expressed corrected for urinary creatinine concentration and specific gravity as both these forms of correction are commonly used in occupational medicine. Only subjects exposed to cadmium who gave five urine collections within the time bands for a single day and whose urine samples had creatinine concentrations $>3 \mathrm{mmol} / \mathrm{l}$ were statistically analysed. Five subjects exposed to cadmium met these criteria and four of five had evidence of low molecular weight proteinuria. Three subjects failed the criteria because of dilute samples, a 
Table 1 Definition of intraindividual variation, acceptable analytical imprecision, and measured analytical imprecison for urinary cadmium expressed as uncorrected and corrected

\begin{tabular}{|c|c|c|c|}
\hline Urinary cadmium & $\begin{array}{l}\text { Uncorrected results \% } \\
\text { (nmol/l) }\end{array}$ & $\begin{array}{l}\text { Creatinine corrected \% } \\
\text { ( } \mu \text { mol/mol creatinine) }\end{array}$ & $\begin{array}{l}\text { Specific gravity corrected \% } \\
\text { (nmol/l corrected to SG } 1.016)\end{array}$ \\
\hline Mean intraindividual biological variation $\left(\mathrm{CV}_{\mathrm{I}}\right)$ & 35.5 & 18.3 & 23.6 \\
\hline Maximum acceptable analytical imprecision $\left(0.5^{\star} \mathrm{CV}_{\mathrm{I}}\right)$ & 17.7 & 9.2 & 11.8 \\
\hline $\begin{array}{l}\text { Analytical coefficient of variation }\left(\mathrm{CV}_{\mathrm{A}}\right) \text { of precision calculated } \\
\text { from duplicate measurements }\end{array}$ & 63 & 87 & 110 \\
\hline Analytical precision calculated from quality control measurements & $\begin{array}{l}3.6 \text { at cadmium concentration } \\
\text { of } 52 \mathrm{nmol} / 1\end{array}$ & $4.1^{\star}$ & $4.0^{\star}$ \\
\hline
\end{tabular}

* Denotes the precision calculated from combination of cadmium and creatinine or specific gravity measurements.

further two subjects had missed a time point during this single day.

For cadmium there was no significant difference $(p>0.05)$ between time points or trend over the time points suggesting no discernible diurnal variation. For urinary RBP there was some significant evidence of a decreasing trend over the day $(p=0.015$ for the specific gravity correction; $p=0.05$ for creatinine correction). However, the urine samples from the second time point were also found to be significantly higher than not only the fourth and fifth time points $(p<0.05)$ but also the first time point for $\mathrm{RBP}$ corrected for specific gravity. This pattern was also reflected, but to a lesser extent, in RBP corrected for creatinine. Therefore the evidence for a decreasing diurnal trend from early morning to mid-afternoon for RBP is not wholly consistent.

On this limited study there is little evidence for suggesting that time of collection may influence the results. Therefore all urine analyses with creatinine concentrations $>3 \mathrm{mmol} / 1$ from the 10 subjects exposed to cadmium were used to calculate intraindividual variation $\mathrm{A}$ maximum number of nine spot urine samples per person were collected over the five day period. Some people provided fewer samples and 74 urine samples in total were involved. In the 10 subjects exposed to cadmium the mean value for urinary cadmium (grand means) expressed as creatinine corrected and specific gravity corrected were respectively $10.73 \mu \mathrm{mol} /$ mol creatinine and $134.9 \mathrm{nmol} / 1$ corrected to specific gravity 1.016 . When necessary statistical analysis used weighted averages to take account of the differences in numbers of urine samples collected per person. Statistical analyses ${ }^{7}$ were used to calculate intraindividual biological variation $\left(\sigma_{\mathrm{I}}\right)$ for cadmium results expressed uncorrected, and corrected for both creatinine and specific gravity. The laboratory analytical variance $\left(\sigma_{\mathrm{A}}{ }^{2}\right)$ was calculated from the paired duplicate analyses of each urine sample. Weighted average within subject total variance $\left(\sigma_{\mathrm{T}}{ }^{2}\right)$ for the subjects exposed to cadmium was calculated with the mean of the duplicate cadmium analyses at each time point. The mean intraindividual biological variance $\left(\sigma_{\mathrm{I}}^{2}\right)$ was calculated according to the formula:

$$
\left(\sigma_{\mathrm{I}}^{2}\right)=\left(\sigma_{\mathrm{T}}^{2}\right)-\left(\sigma_{\mathrm{A}}^{2} / 2\right) \text {. }
$$

The intraindividual biological coefficient of variation $\left(\mathrm{CV}_{\mathrm{I}}\right)$ was calculated as:

$\mathrm{CV}_{\mathrm{I}}=\left(\sigma_{\mathrm{I}} /\right.$ (grand mean of 74 samples $\left.)\right) \times 100$.

The coefficient of analytical variation $\left(\mathrm{CV}_{\mathrm{A}}\right)$ calculated from the paired analyses was defined as:

$\mathrm{CV}_{\mathrm{A}}=\left(\sigma_{\mathrm{A}} /\right.$ (grand mean of 74 samples $\left.)\right) \times 100$.

In clinical medicine it has been suggested that the acceptable precision of a method to measure an analyte should be less than half the average intraindividual biological variation $\left(<\left(\mathrm{CV}_{\mathrm{I}}\right) / 2\right.$, table 1$) .^{7}$ Cadmium data in the laboratory volunteers was not included in this analysis due to the low concentrations of cadmium measured in these subjects.

Table 2 shows the results from a similar statistical analysis for urinary RBP. The 15 subjects (10 exposed to cadmium and five non-exposed) were separated into normal and those with abnormal low molecular weight proteinuria according to their mean RBP compared with the laboratory reference range. Nine subjects had abnormal proteinuria and six subjects had normal proteinuria; separate statistical analyses were performed on each subgroup.

The use of creatinine correction reduced the mean intraindividual variation $\left(\mathrm{CV}_{\mathrm{I}}\right)$ for urinary cadmium to half the level for cadmium results expressed uncorrected. For RBP the reduction in $C_{\mathrm{I}}$ with creatinine correction was not as pronounced. The acceptable analytical precison for urinary cadmium and creatinine measurements combined is $9.2 \%$. For RBP the similar value is $20 \%$ derived from subjects with

Table 2 Definition of intraindividual variation, acceptable analytical imprecision, and measured analytical imprecison for urinary retinol binding protein expressed as uncorrected and corrected (results shown for subjects with abnormal and normal renal function)

\begin{tabular}{|c|c|c|c|}
\hline & $\begin{array}{l}\text { Uncorrected results } \\
\%(\mu \mathrm{g} / \mathrm{l})\end{array}$ & $\begin{array}{l}\text { Creatinine corrected } \\
\%(\text { mg/mol creatinine })\end{array}$ & $\begin{array}{l}\text { Specific gravity corrected } \\
\%(\mu \mathrm{g} / \mathrm{SG} 1.016)\end{array}$ \\
\hline \multicolumn{4}{|l|}{ Retinol binding protein in abnormal group $(n=9)$ : } \\
\hline Mean intraindividual biological variation $\left(\mathrm{CV}_{\mathrm{I}}\right)$ & 48.2 & 40.1 & 41.4 \\
\hline Maximum acceptable analytical precision & 24.1 & 20.0 & 20.2 \\
\hline Analytical precison $\left(\mathrm{CV}_{\mathrm{A}}\right)$ calculated from duplicate measurements & 13.8 mean $=5959 \mu \mathrm{g} / 1$ & 11.0 & 13.3 \\
\hline Analytical precision calculated for quality control measurements & 9.7 at $179 \mu \mathrm{g} / 1$ & $9.9^{\star}$ & $9.8^{\star}$ \\
\hline \multicolumn{4}{|l|}{ Retinol binding protein in normal group $(n=6)$ : } \\
\hline Mean intraindividual biological variation $\left(\mathrm{CV}_{\mathrm{I}}\right)$ & 35.6 & 30.6 & 30.3 \\
\hline Maximum acceptable analytical precision & 17.8 & 15.3 & 15.1 \\
\hline Analytical precison $\left(\mathrm{CV}_{\mathrm{A}}\right)$ calculated from duplicate measurements & 13.9 mean $=154 \mu \mathrm{g} / 1$ & 13.2 & 13.9 \\
\hline Analytical precision calculated for quality control measurements & 9.7 at $179 \mu \mathrm{g} / \mathrm{l}$ & $9.9^{\star}$ & $9.8^{\star}$ \\
\hline
\end{tabular}

^ Denotes the precision calculated from combination of RBP and creatinine or specific gravity measurements. 
abnormal proteinuria and $15 \%$ from normal subjects. It is interesting to note that particularly for cadmium measurements the analytical precision derived from duplicate analyses of subjects' urine was worse than that calculated from repeated measurement throughout this study of reconstituted, lyophilised quality control material. This may reflect the ideal characteristics of this material, and may not reflect the true analytical precision when applied to real life monitoring of samples which have been frozen or have urinary precipitate.

Both the intraindividual biological variation and analytical precision of the methods used influence the ability to detect significant changes in the excretion of an analyte in a person. The estimation of a significant difference $(\mathrm{p}<0.05)$ between two successive measurements in a population is given by the formula:

$$
1.96 \times(\sqrt{ } 2) \times \mathrm{CV}_{\mathrm{T}}
$$

where $\mathrm{CV}_{\mathrm{T}}$ is defined as $\mathrm{V}\left(\mathrm{CV}_{\mathrm{A}}{ }^{2}+\mathrm{CV}_{\mathrm{I}}^{2}\right)$. Mean $\mathrm{CV}_{\mathrm{T}}$ is calculated in this study as either $20.2 \%$ or $18.8 \%$ for creatinine corrected cadmium results, depending on the defined analytical variation $\left(\mathrm{CV}_{\mathrm{A}}\right)$ from duplicate measurements $\left(\sqrt{ }\left(8.7^{2}+18.3^{2}\right)\right.$ or quality control data $\left(V\left(4.1^{2}+18.3^{2}\right)\right.$. Thus, if in this small study the calculated mean $\mathrm{CV}_{\mathrm{T}}$ is representative of that found in a large population, the minimum difference between two results:

$$
\begin{aligned}
& (100 \times(\mid \text { result2-result1 } \mid) / \\
& ((\text { result } 1+\text { result } 2) / 2))
\end{aligned}
$$

which is statistically different at the $95 \%$ level of certainty is calculated as about $54 \%$ with our mean value for the analytical variation. From the data in this study, a urinary cadmium measurement of $10 \mu \mathrm{mol} / \mathrm{mol}$ creatinine could still not be called significantly different from a subsequent urine sample taken in a short time frame from the same person measured as 17.4 $\mu \mathrm{mol} / \mathrm{mol}$ creatinine.

A similar statistical analysis was performed for urinary RBP. With the defined mean $\mathrm{CV}_{\mathrm{I}}$ and Health and Safety Laboratory's analytical precision of about $12 \%$ (combined RBP and creatinine), we calculate that a difference of about $>110 \%$ would be necessary between two successive creatinine corrected RBP results to be identified as showing a significant change $(\mathrm{p}<0.05)$ in a person with abnormal proteinuria. Similar analysis gives slightly smaller percentage differences for subjects without renal damage.

Figures 1 and 2 show the relation between long term laboratory analytical precision and percentage differences between successive measurements for creatinine corrected urinary cadmium and retinol binding protein that can be described as being significant $(p<0.05)$. These graphs can be used to aid interpretation of results measured by different laboratories with varying analytical precisions.

\section{Discussion}

The use of corrections for creatinine or specific gravity for analytes in random, untimed urine samples is widely used in occupational medi-

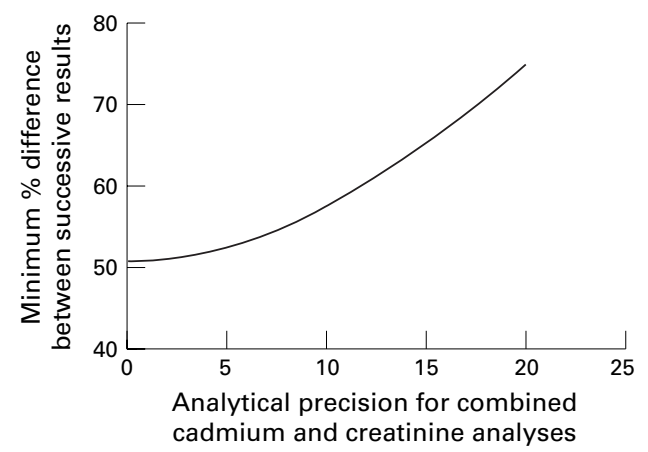

Figure 1 Relation between analytical precision $\left(C V_{A}\right)$ and interpretation of the significant percentage difference* $(p<0.05)$ between successive spot urine samples for cadmium results corrected for creatinine. ${ }^{\star}(100 \times$ $(\mid$ result $2-$ result $1 \mid) /(($ result $1+$ result 2$) / 2))$.

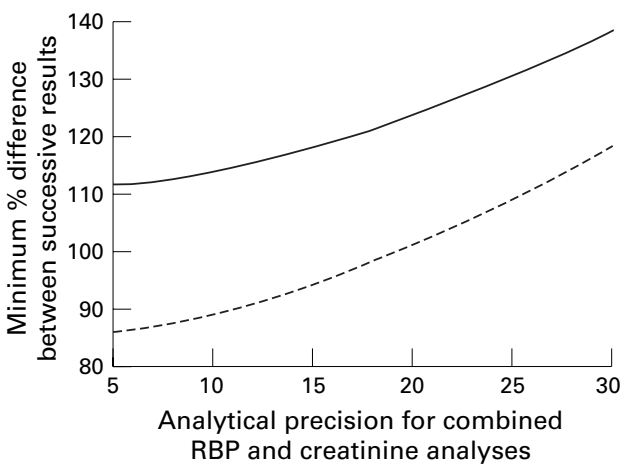

Figure 2 Relation between laboratory analytical precision and interpretation of the significant percentage difference* $(p<0.05)$ between successive spot urine samples for $R B P$ results corrected for creatinine. The unbroken line is derived from subjects with abnormal proteinuria; the broken line is derived from normal subjects. * $(100 \times$

$(\mid$ result $2-$ result $1 \mid) /(($ result $1+$ result 2$) / 2))$.

cine. However, the use of creatinine correction in general has not been without criticism by some authors, mostly questioning the degree of variability of creatinine excretion over time in a person or as accurately reflecting urinary flow rate. ${ }^{11}{ }^{11}$ Because of the physiological mechanism of renal excretion of some analytes, the use of creatinine correction may indeed be inappropriate. Therefore the use of correction methods with creatinine or specific gravity have to be considered on an analyte by analyte basis.

Studies have been performed comparing the value of such corrected urine data from random, untimed samples against analyte, including cadmium excretion derived from timed urine collections. ${ }^{12-14}$ Such studies have often reached opposing conclusions. Thus Trevisan $e t a l^{13}$ reported that, based on the correlations between spot and timed urine collections in a cohort study, creatinine correction of cadmium concentrations in spot urine samples was more suitable for biological monitoring. In contrast, Alessio et $a l^{14}$ found no advantage in correcting for creatinine or specific gravity. The difficulties in the accurate collection of a timed urine collection are well understood in the clinical setting, and such difficulties are magnified in the occupational setting. Therefore the investigation of the value of methods for correction of spot urinary analyte concentra- 
tions as a practical measure of expressing true analyte excretion are valuable to occupational and environmental medicine. This report studies the effect of urine correction on the within person variation of excretion, with repeat measurements in a person rather than the correlation between spot and timed urine concentrations from a cohort.

The study compared the intraindividual variation of urinary cadmium and RBP in multiple samples collected over the short time frame of a working week from subjects with high historical, but no current, occupational exposure to cadmium. Reduction in variability for a person with correction for creatinine or specific gravity implies that the corrected value is more likely to reflect the true excretion of cadmium or RBP in that person. The application of standard clinical laboratory techniques ${ }^{67}$ for validating a diagnostic test to the biomarkers in this study allows definition of useful variables for the occupational health professional and laboratory staff. These variables include the appropriateness of correction for creatinine or specific gravity, the acceptable analytical precision necessary to gain best interpretable results, the uncertainty associated with a single urinary cadmium and RBP measurment, and the difference between serial measurements which can be deemed significant and the influence of analytical precision on this value.

There was no clear evidence of a diurnal variation for cadmium or RBP results corrected for specific gravity or creatinine over the periods studied in a few of the workers exposed to cadmium. The variability in the RBP results from period to period probably reflects the large intraindividual biological variation for RBP (about 40\%). The data suggest that if there is a diurnal variation it is undetectable compared with the other sources of variation.

The intraindividual biological variation in urinary cadmium and RBP is smaller for results corrected for creatinine and specific gravity than for uncorrected results. The influence of the urinary correction on reducing intraindividual variation is greater for cadmium than RBP. For urinary cadmium analyses the uncorrected intraindividual biological variation was reduced by $50 \%$ by correction for creatinine, which seemed marginally better than correction for specific gravity. Thus correction for creatinine or specific gravity of spot urine samples for monitoring subjects exposed to cadmium seems appropriate. The data from this study suggest that laboratories undertaking analyses for cadmium monitoring should have real long term method precisions of less than $9 \%$ and $15 \%$ for the combined analytical precision of urinary cadmium and RBP respectively when corrected for creatinine.

The data from this short term study are compatible with our long term findings from quarterly biological monitoring over a five year period in a similar cohort of workers with high historical exposure but no exposure over the observation period (unpublished data). The mean measured coefficients of variation $\left(\mathrm{CV}_{\mathrm{T}}\right)$ in each person over the five year period were $28 \%$ and $56 \%$ for urinary cadmium and RBP respectively, expressed when corrected for creatinine. The equivalent coefficients of variation reported in this short term study are $20 \%$ and $42 \%$. The higher coefficents of variation measured over the five year period probably reflect higher analytical variation over that time scale calculated from long term quality control data, rather than the influences of decreases in kidney burden of cadmium or changes in renal function.

The 95\% CI associated with a single measurement in a person can be described by the measurement value $\pm 2 \times \mathrm{CV}_{\mathrm{T}}$, which depends on both biological and analytical variation. For example, with our data presented in this report of a $\mathrm{CV}_{\mathrm{T}}$ of $20 \%$ for urinary cadmium results corrected for creatinine, a true excretion of $5 \mu \mathrm{mol} / \mathrm{mol}$ creatinine would have a $95 \%$ CI of $5 \pm 2 \mu \mathrm{mol} / \mathrm{mol}$ creatinine. This implies that in the same person within a short time frame and no change in exposure or cadmium body burden, there is a $95 \%$ chance that other spot urine results would fall between 3 and $7 \mu \mathrm{mol} / \mathrm{mol}$ creatinine.

Figures 1 and 2 can be used to define minimum significant differences betweeen consecutive measurements of urinary cadmium and RBP for different levels of laboratory analytical precision. Our calculated significant differences between successive results for urinary cadmium of $>54 \%$ suggest that to determine the presence or absence of a real trend in increasing urinary cadmium excretion, implying increased cadmium absorption, may require quarterly measurements as a practical monitoring policy. Such a strategy would be a pragmatic balance between the cost of increased frequency of monitoring against better definition of trends in body burden of cadmium. Similarly our significant difference of $>110 \%$ between successive RBP measurements would suggest that in subjects with evidence of low molecular weight proteinuria, caution should be applied about overinterpretation of a single result as signifying an improvement or worsening of the condition. However, our experience and data from unexposed subjects in this study suggest that against a properly defined normal range, false positive results in RBP corrected for creatinine from random spot urine normal samples are rare.

In epidemiological studies in which trends are discerned from populations, the importance of uncertainty associated with a single result in a person is reduced by the increasing cohort size. In occupational health and health surveillance, interpretation of a person's results is important. The approach used in this paper may be applicable to other analytes with relatively long half lives in the body. Other approaches may need to be used for analytes with short half lives. However, we think that the definition of the uncertainty or error associated with any measurement for biological monitoring can only help to improve the value of such measurements.

1 Kjellstrom T, Nordberg GF. A kinetic model of cadmium metabolism in the human being. Environ Res 1978;16:248- 
2 Roels H, Lauwerys R, Buchet J, et al. Health significance of cadmium induced renal dysfunction: a five year follow up. $\mathrm{Br}$ F Ind Med 1989;46:755-64.

3 Hartley C, Mason HJ, Chettle D, et al. Occupational cadmium exposure and glomerular filtration rate effects. $\mathcal{F}$ Occup Med Toxicol 1992;1:337-47.

4 Lauwerys R, Bernard A, Buchet J, et al. Assessment of the health impact of environmental exposure to cadmium: contribution of epidemiologic studies carried out in Belgium. Environ Res 1993;63:200-6.

5 Lauwerys R, Roels H, Riegners M, et al. Significance of cadmium concentration in blood and urine in workers exposed to cadmium. Environ Res 1979;20:375-91.

6 Elevitch F, ed. 1976 Aspen Conference on Analytical Goals in Clinical Chemistry. Skokie, IL: College of American Pathologists, 1977.

7 Fraser CG, Harris EK. Generation and application of data on biological variation. Crit Rev Clin Lab Sci 1989;27:409-37.

8 Mason HJ, Calder IM. The correction of urinary mercury concentration samples. Occup Environ Med 1994:51:287.
9 Ellis KJ, Cohn SH, Smith TJ. Cadmium inhalation exposure estimates: their significance with respect to kidney and liver cadmium burden. F Toxicol Environ Health 1985;15:173-87.

10 Boehniger MF, Lowry LK, Rosenberg J. Interpretation of urine results used to assess chemical exposure with emphasis on creatinine adjustments: a review. Am Ind Hyg Assoc 7 1993;54:615-27.

11 Greenberg GN, Levine RJ. Urinary creatinine excretion is not stable: a new method for assessing urinary toxic substance concentrations. f Occup Med 1989;31:832-8.

12 Berlin A, Alessio L, DEll'Orto A, et al. Problems concerning the usefulness of adjustment of urinary cadmium for creatinine and specific gravity. Int Arch Occup Environ Health 1985;55:107-11.

13 Trevisan A, Nicoletto G, Maso S, et al. Biological monitoring of cadmium exposure: reliability of spot urine samples. Int Arch Occup Environ Health 1994;65:373-5.

14 Alessio L, Apostoli P, Forni A, et al. Biological monitoring of cadmium exposure - an Italian experience. Scand 7 Work Environ Health 1993;19(suppl 1):27-33.

\section{Rejected manuscripts}

From February 1994, authors whose submitted articles are rejected will be advised of the decision and one copy of the article, together with any reviewer's comments, will be returned to them. The fournal will destroy remaining copies of the article but correspondence and reviewers' comments will be kept. 